\title{
DIRECT VENEERS - FROM DESICN TO IMPLEMENTATION
}

Zoltán Miskolczy ${ }^{1 \mathrm{a}^{*}}$, Barbara Kispélyi ${ }^{1 \mathrm{~b}}$, Judit Borbély ${ }^{1 \mathrm{c}}$, Péter Hermann ${ }^{1 \mathrm{~d}}$

'Department of Prosthodontics, Faculty of Dentistry, Semmelweis University, Budapest, Hungary

${ }^{\mathrm{a}} \mathrm{DMD}$, clinical dentist

${ }^{b} \mathrm{DMD}, \mathrm{PhD}$, Associate Professor

'DMD, PhD, Associate Professor

dDMD, MSc, PhD, Professor, head, Vice-Rector

Cite this article:

Cite this article: Miskolczy Z, Kispélyi B, Borbély J, Hermann P. Direct veneers - From design to

implementation. Stoma Edu J.2016; 3(1-2):33-38.

ABSTRACT https://doi.org/10.25241/stomaeduj.2016.3(1-2).art.5

Aim: In a 58-year old female patient, the frontal teeth damaged by attrition were reconstructed by direct veneer technology. Frontal tooth configuration and esthetical appearance were restored. Using this case as an example, we describe the key steps of producing a direct veneer and study the scope of application of this technique and the expected lifetime of reconstructed structures. Summary: The adhesive technique makes it possible to esthetically optimize such teeth which show no decay lesions without doing any damage. This way, we can also satisfy patients, who refrain from any more invasive treatment, e.g. crowning. Based on the literature data shown, it can be stated that with appropriate indications - direct veneer restorations can be equivalent alternatives to conventional "golden standard" restorations.

Key learning points: 1 . With the development of the adhesive technique, minimally invasive and noninvasive procedures play a bigger and bigger role in esthetic dentistry beside conventional interventions. 2. Our goal was to restore a harmonious smile, taking into account the principles of Visagismo. 3. Sufficient bonding power can only be obtained on the enamel surface, so it is reasonable to consider the indications of direct veneer if a large surface of dentin is exposed.

Keywords: direct veneers, smile design, composite layering, diagnostic wax-up, composite polishing.

\section{Introduction}

Beauty is a term used more and more often in our everyday life. There is nothing new about it: humans have shown great concern about their own appearance since ancient times. "Ideal beauty" is admired because it features characteristics that, in that particular culture, are associated with perfection. According to the Ancient Greek saying beauty is in the eye of the beholder, therefore there are differences in what the individual people find beautiful. Plato considered beauty to be the idea (shape) that lies above all ideas. Smile plays a key role in the beauty and esthetics of the human face. It is an accord of the mimic facial muscles, which extends even to the area of the eyes. Humans generally consider smile a sign of dearness, happiness and joyfulness, and it also radiates self-confidence and trustworthiness. Teeth are the most important elements of the overall picture of a smile. Therefore, it is not difficult to understand that there can be a correlation between the esthetic problems of one's teeth and his/her mental and social issues. It is the duty of the dentist with an interdisciplinary approach to identify the problem, to get to know the patient to such an extent sufficient for the treatment and to provide the complex treatment itself, with appropriate communication an important part of the latter. It can be clearly stated that the procedures used in dentistry have undergone significant development for the last years. With the development of the adhesive technique, minimally invasive and noninvasive procedures play a bigger and bigger role in esthetic dentistry beside conventional interventions.

Indications for direct veneers can be different abnormities in terms of color, shape or position. Below is an incomplete list of cases that might call for such restorations:

\section{Color differences:}

- Hypocalcification

- Hypoplasia

- Fluorosis

- Tetracycline damage

- Discoloration (not removable by scaling and tooth whitening)

- Discoloring effects of earlier amalgam fillings

- Age-related physiological discoloration 


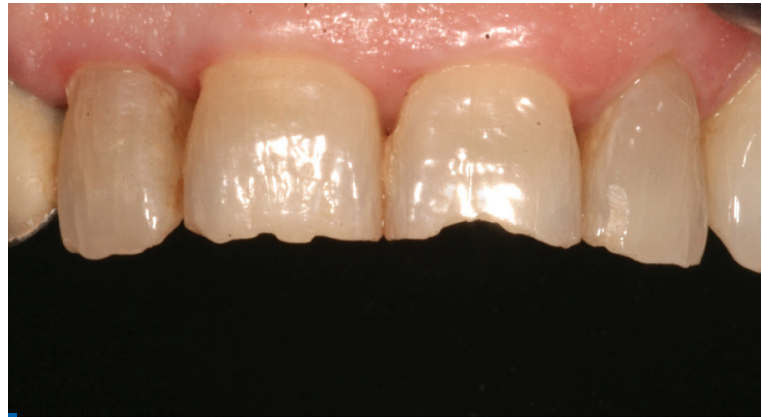

Figure 1.58 - y.o. woman visiting the clinic with frontal teeth damaged by attrition

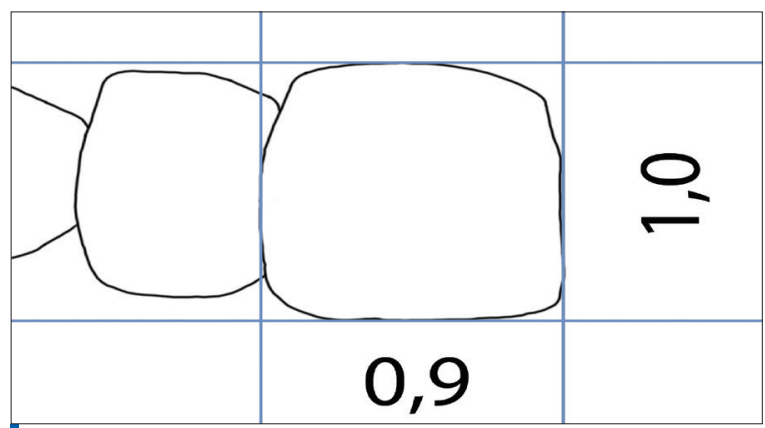

Figure 3 . In young patients, tooth width is generally $65 \%$ of crown length. In older age, this ratio becomes generally $90 \%$ - this is what we considered in the design phase

\section{Shape differences:}

- Asymmetry between the two sides

- Fractured teeth due to trauma

- Abrasion

- Attrition

- Erosion

- Peg lateral incisors

- Restoration of teeth replacing missing teeth by means of orthodontics

\section{Positional abnormities:}

- Ectopic teeth

- Disharmony in the incisal curvature

- Diastema closure

- Anomalies in the inclination of teeth

- Torsion

- Infraocclusion

- Centerline shift

Nowadays it is obvious that we might design the patients' smiles with different characters. According to Rosenstiel et al. gender, ethnic, age group etc. determines people's thinking about the aesthetic smile character. Yet, there are people who cannot distinguish two different smile characters. ${ }^{1}$ This points out the dentist's responsibility to carefully design and provide the patient with different alternatives. Paolucci et al. worked out the theory of Visagismo, which is a new approach to direct veneer design. ${ }^{2}$ In their opinion, the dissatisfaction of patients - which occurs so often in the clinical practice - is caused by the disharmony between the new smile created and the personality of the patient. Visagismo is a Portuguese word that means face shaping by

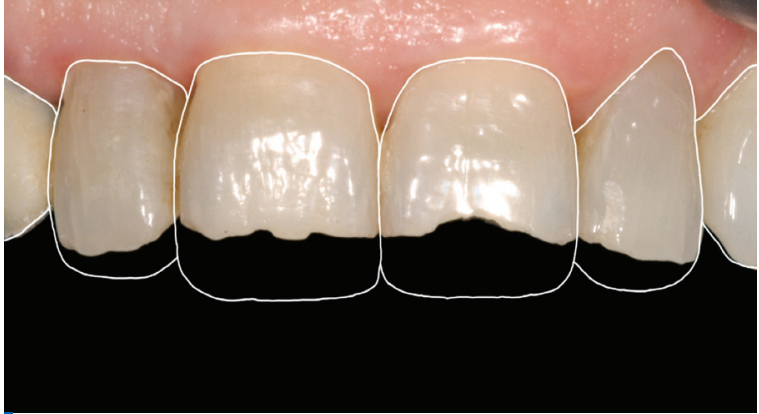

Figure 2. We selected a square tooth shape that matches "phlegmatic" temperament as a basis of further design

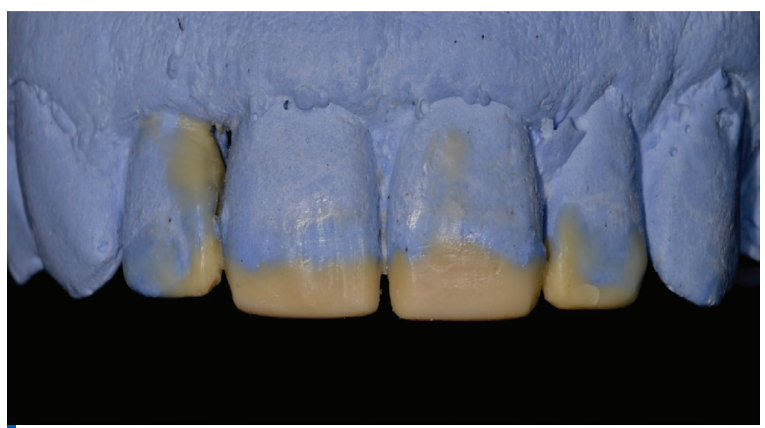

Figure 4. Diagnostic wax-up

means of makeup. Visagismo is a guideline for dentists and dental technologists, which helps create restorations which combine esthetical appearance with psycho-social characteristics. A personalized treatment using these guidelines may result in changes in the patient's behavior, posture and speech, too. ${ }^{2}$ What does all this mean in the dentists' practice? To answer this question, it is inevitable to define Hippocrates' four temperaments (personality types). The first is choleric/strong: assertive, objective, vigorous and passionate. The second is sanguine/dynamic: hearty, open-minded, talkative, joyful, brimming and enthusiastic. The third is melancholic/ emotion-oriented: organized, perfectionist, restrained, shy. The fourth is phlegmatic/peaceful: tactful, non-violent, mysterious, idealistic, and prone to apathy and conformity. Paolucci et al. defined the characteristics of the teeth matching each temperament as follows ${ }^{2}$ :

Choleric/strong: Rectangular incisors, dominant central incisors, flat incisal edges, sharp cusps, vertical longitudinal axes;

Sanguine/dynamic: Triangular incisors, upwardarching smile line, converging tooth angles, inclined cusps;

Melancholic/emotion-oriented: Oval incisors, dominant central incisors, rounded cusps, delicate lateral incisors, round frontal tooth arch;

Phlegmatic/peaceful: Square incisors, lack of dominance, divergent tooth axes, horizontal configuration. 2,3 The above-mentioned features show that it may be necessary to learn the patient's personality in order to set up a complex esthetical treatment plan. 


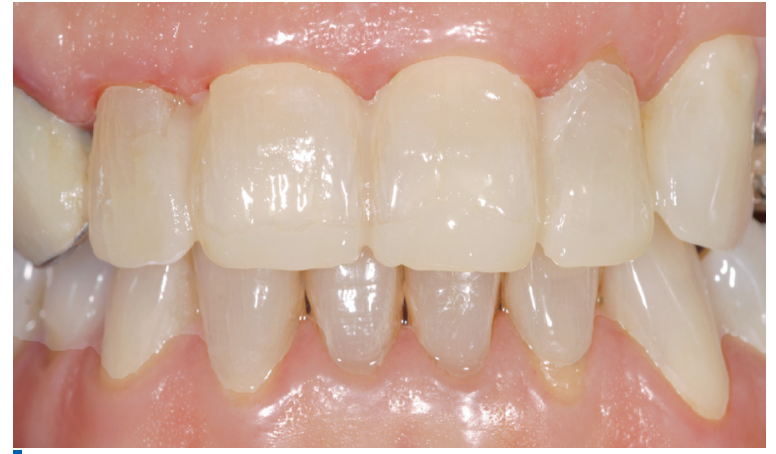

Figure 5. After creating the mock-up, it is possible to do corrections intraorally upon the patient's request

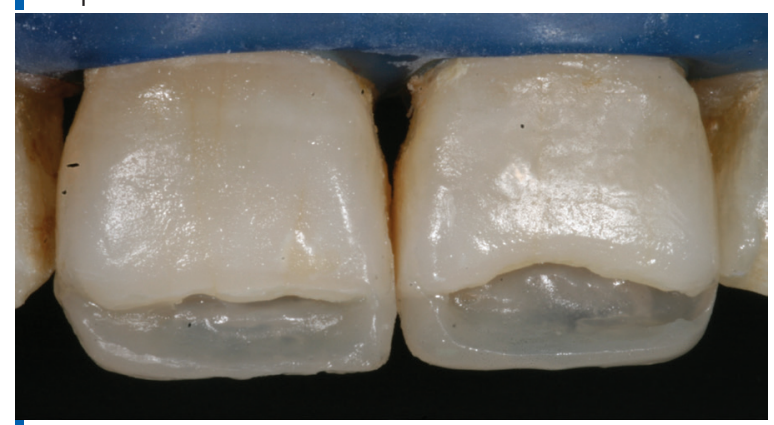

Figure 7. The completed palatal enamel

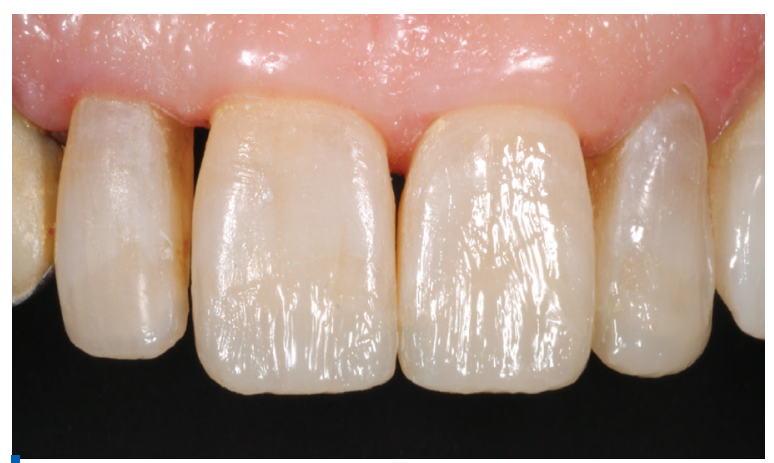

Figure 9. Restoration after applying the enamel layer

\section{Case report}

In our example case, the patient was a 58-year old female, who visited the clinic with frontal teeth damaged by attrition. She had a good and stable periodontal situation, did not smoke and had no other illnesses. In terms of functional pathology, no abnormities were found, therefore no other treatment was necessary. We assume that abrasion had been caused by a previous (already removed) bridge in the lateral region and bruxism induced by it (Fig. 1). Our goal was to restore a harmonious smile, taking into account the principles of Visagismo. Based on intensive communication with the patient, we managed to find a balance between her requirements and what was feasible. After long talks with her, we chose the square tooth shape, being characteristic of a primarily "phlegmatic" temperament, as the basis of the further stages of the design process (Fig. 2).

It was inevitable to take the patient's age into

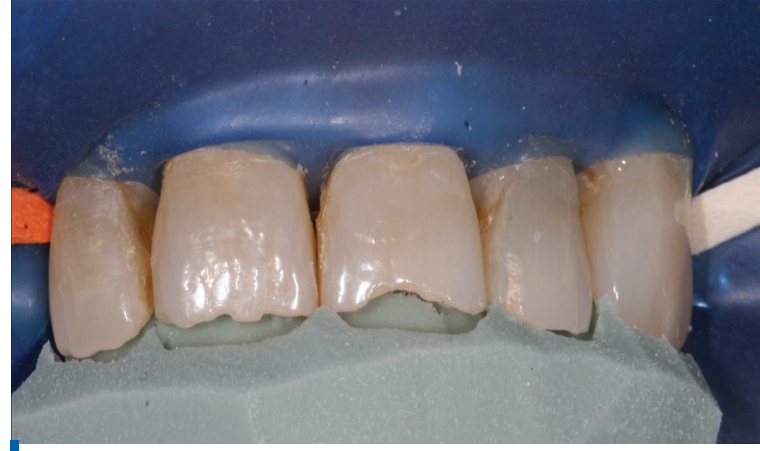

Figure 6. Enamel prepared off to 45 degrees and the silicon key

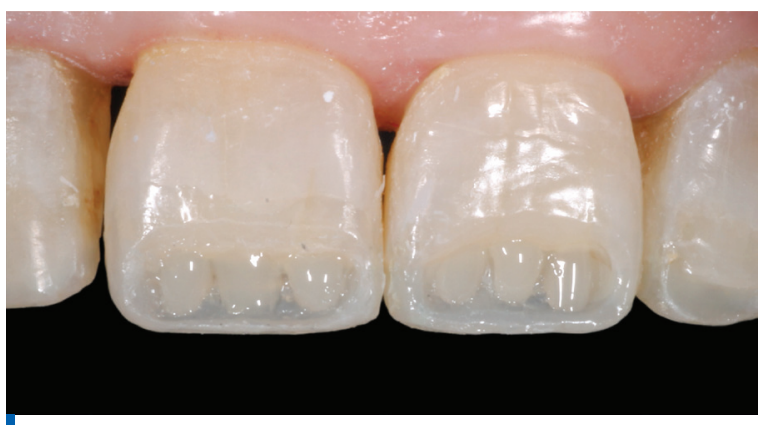

Figure 8. Upon the patient's request, we wanted to give the frontal teeth a youthful characters and that's how we applied the dentin color shade, too.

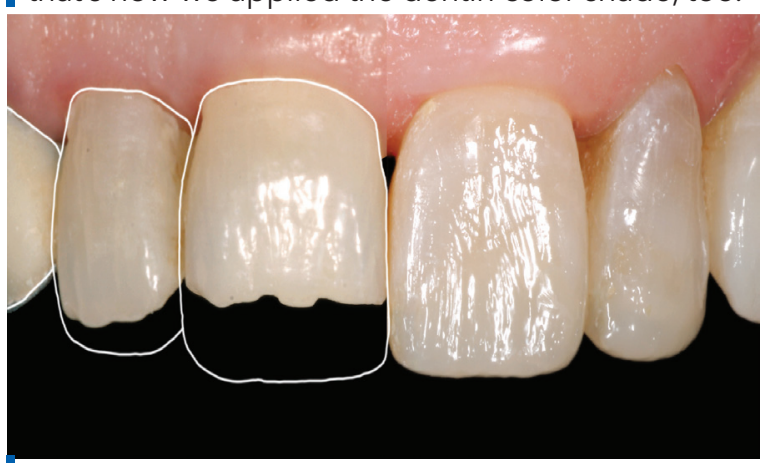

Figure 10. The character of the teeth matches the requirements of the original plan

account. Younger patients' smiles are generally characterized by longer, rectangle-shaped central incisors, more marked edges, mamelons, HALO effects, visible perikymata and surface grooves, brighter colors, etc. (Fig. 3) These characteristics are different in older patients. Incisors tend to be shorter, and individual features (edge transparency, mamelons, HALO effect, perikymata, etc.) are significantly less or not at all visible on the teeth. ${ }^{4}$ The diagnostic wax-up was produced by the dental technician with these in mind and computer-aided design. The visualization plan created was discussed with the patient in detail and we outlined the options to her. After the diagnostic wax-up, the silicone impression key was created in order to manufacture the intraoral mock-up (Fig. 4). ${ }^{5}$

During the next session, the mock-up was created, which we used to demonstrate to the patient her later treatment options. By re-shaping the mock- 


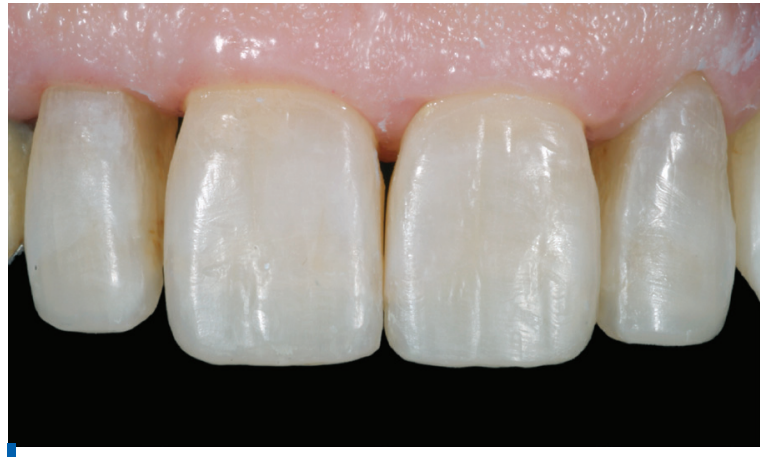

Figure 11. The completed restoration

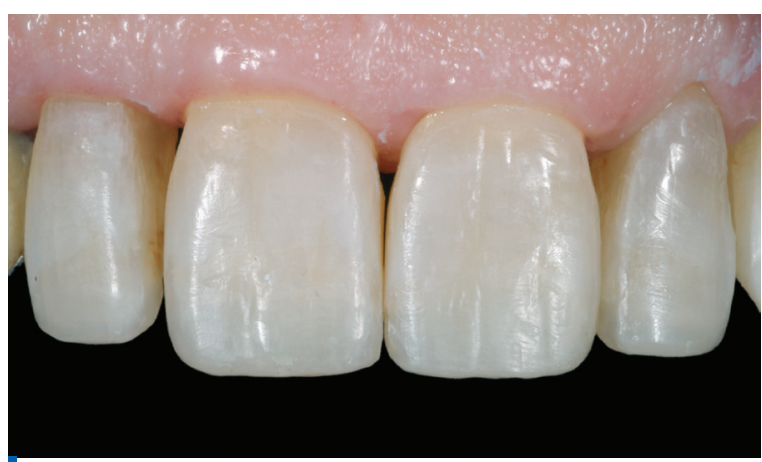

Figure 13. Six-month checkup. Teeth 21, 22 showed that the closure line at the edge had become minimally visible

up, it was possible to finalize the planned shape. (Fig. 5)

\subsection{Preparing the teeth}

After obtaining the desired shape, we prepared a silicon key intraorally for the final restoration. Thereafter, we carefully removed the composite material used for the mock-up. We chose the A3 tooth shade by using Vita Classic shade guide. We put - whitout adhesive technique - a minimal amount of the appropiate dentin and enamel on the tooth, which were removed after reviewing the shade. After that we prepared the enamel edges off to 45 degrees (Fig. 6). An important step is to select the appropriate tooth color shade before isolating, before the tooth material dehydrates. Thereafter, the dental rubber dam, which is essential in such restoration works, is put into place. We conditioned the surface by means of total etch technology - 37\% orthophosphoric acid for 40 seconds, Adper Single Bond 2 Adhesive (3M ESPE, St. Paul, MN 55144-1000, USA). ${ }^{6}$

\subsection{The composite layering technique}

The first step was to put the palatal enamel to teeth upper right and upper left central and lateral incisor (Filtek Ultimate A3 Enamel, 3M ESPE, USA). We used a vertically cut silicon block in the process (Fig. 7). Upon to the patient's request, we intended to give a youthful character to the frontal teeth - we applied the dentin color shade accordingly (Filtek Ultimate A3 Dentine, 3M ESPE, USA). ${ }^{7}$ We believed that any more marked edge transparency or HALO effect was not necessary, so we could proceed to the application of buccal enamel (Fig. 8). ${ }^{8}$ During

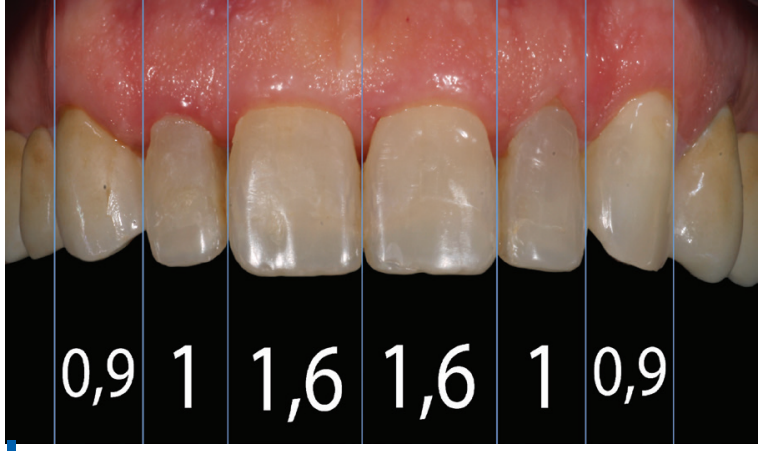

Figure 12. The relative widths of the teeth can be evaluated. It is visible that the ratio of the large and small incisors complies with the "golden standard" and the relative width of the two canines is larger than the "golden standard"

the application of enamel layers, we used Teflon tape to isolate the individual teeth from each other (Fig. 9). Using a silicon brush (Micerium S.p.A, Avegno (Ge), Italy) was a great help for us in working out the surface. The sticking of the brush can further be reduced using Composite Primer (GC, Tokyo, Japan). ${ }^{9}$ Special care must be taken not to break the morphologic elements of existing tooth material in the restored part but to "continue" them carefully (Fig. 10). ${ }^{10}$

\subsection{Lifelike shaping of the characters and polishing}

The characters were created in a lifelike way during finishing/polishing. As a first step, we used FG mounted Arkansas stone at 40,000 RPM with water cooling to create the above-mentioned morphologic elements, correct the routing of marginal ridges and create the final shape of the surfaces. To polish approximal area, we used Super-Snap (Shofu Dental Corporation, Kyoto, Japan) disks. ${ }^{11}$ Then, we reworked the surface using FG mounted Arkansas stone at low RPM and 3-micron polishing paste - SuperPolish (Kerr SA, Bioggio, Switzerland). The final polishing was carried out with the appropriate steps of the ENA Shiny polishing system (Micerium S.p.A (Ge), Avegno, Italy). ${ }^{12}$ Studying the complete restoration, the relative widths of the teeth can be evaluated. It can be seen that the ratio of the large and small incisors meets the "golden standard" (Fig. 11). ${ }^{13}$ The relative width of the two canines is larger than the "golden standard" value, but the dominant canines do not have any disconcerting effect on the patient's smile (Fig. 12). ${ }^{14}$ At the six-month checkup, the clinical situation was as follows (Fig. 13). The patient was completely satisfied with the restoration (Fig. 14). At the examination, we found the restoration to be intact; the closure line at the edge had become minimally visible at teeth upper left central and lateral incisor. We removed this by polishing (Super-Snap, Shofu Dental Corporation, Kyoto, Japan) by replacing the upper lateral bridges on both sides, we created an esthetic smile that met the requirements of our patient, too (Fig. 15). 


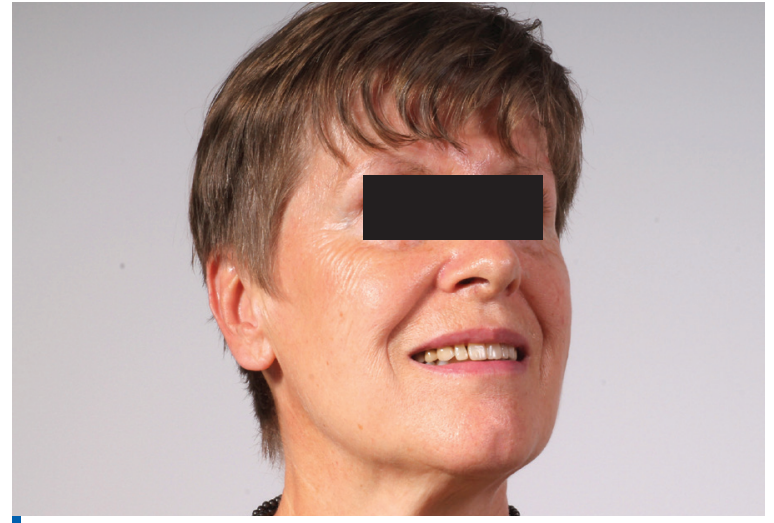

Figure 14. The content smile of the patient

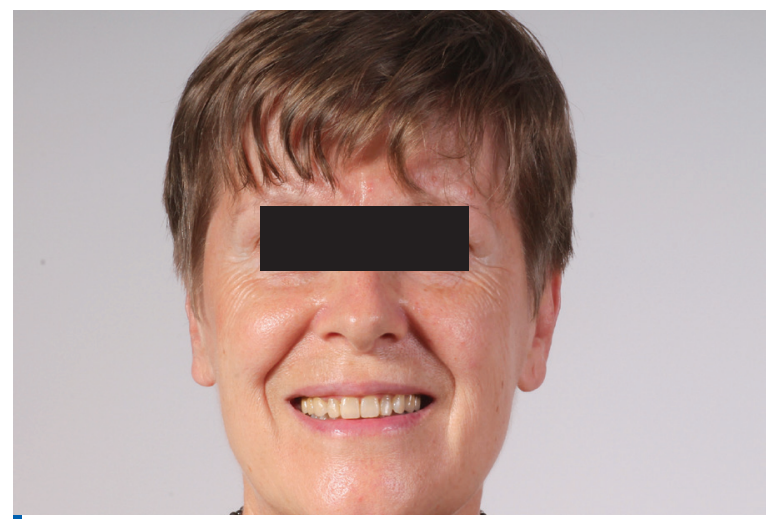

Figure 16. Smile of the patient

\section{Discussion}

Many studies prove the usability of direct veneers in the everyday dental practice. Frese et al. ${ }^{15}$ conducted a five-year follow-up study on 176 direct veneers. All veneers were made without preparatory works in order to provide color and shape correction at the Conservative Dental Clinic of Heidelberg University, Germany. After the follow-up period, the restorations were grouped into three categories: "failure”, "survival" and "success". Damage occurred in 30 cases, but all of them could be repaired, so they belonged to the "surviving" group. In the end, no restoration was classified as "failure". This made the five-year survival rate to be $100 \%$ in this group, whose size can be considered as statistically significant. With the faults taken into account, the success rate was 84.6\%.15 Garcia-Godoy et al. conducted a 6-year follow-up study on in vitro and in vivo restorations. ${ }^{14}$ In vitro, they fitted $\mathrm{MO}$ restoration to 32 extracted human teeth, then exposed them to various influences for 6 years in a randomized way (immersion in water, thermo-mechanical load, both). In the in vivo group, direct restorations were fitted into 68 teeth of 30 individuals in total. After 6 years, the closure at the edges was evaluated. The in vitro experiments showed an 85 to 100-percent success for the enamel, while a 42 to 52 -percent one for the dentin. In the in vivo group, the edge closure was intact in 75 to 90 percent for the enamel.

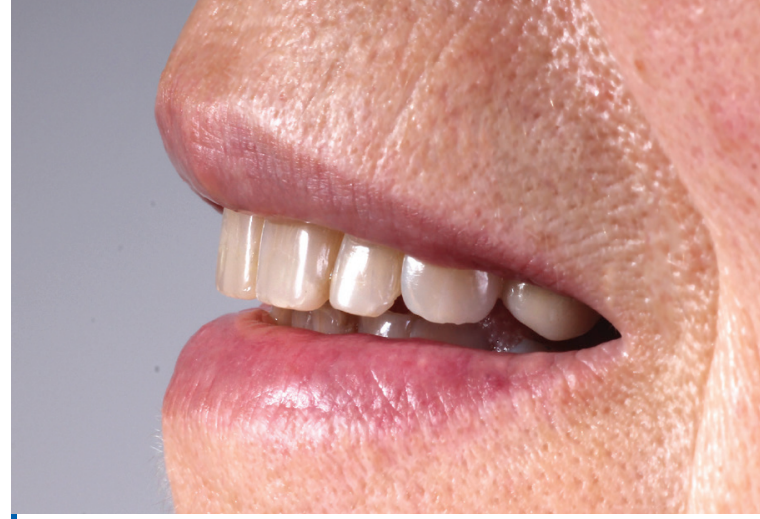

Figure 15. Smile of the patient

It was shown that there were significant differences between the enamel and the dentin. ${ }^{16}$ In a third case, Gresnigt et al. monitored 96 direct veneer restorations for more than 40 months. Survival rate was $87.5 \% .{ }^{17}$ The adhesive technique makes it possible to esthetically optimize such teeth which show no decay lesions without doing any damage. This way, we can also satisfy patients who refrain from any more invasive treatment, e.g. crowning. Based on the literature data shown, it can be stated that - with appropriate indications - direct veneer restorations can be equivalent alternatives to conventional "golden standard" restorations. As Garcia-Godoy et al. showed, the "appropriate indications" are a very important factor, because a sufficient bonding power can only be obtained on the enamel surface, i.e. it is reasonable to consider the indications of direct veneer if a large surface of dentin is exposed. ${ }^{17}$

Our experience justifies the facts presented above. In case there is a sufficent amount of enamel surface we can decide on direct veneer restauration after measuring the possibility of preparing, elaborating and polishing the restauration.

\section{Conclusions}

To conclude, in the first place we can say that this adhesive technique makes it possible to esthetically optimize such teeth which show no decay lesions without doing any damage. We are also in a position to say that the facts presented in this paper are justified and supported by our experience.

Secondly, another conclusion would be that, if there is a sufficent amount of enamel surface, a decision that could be taken would be to resort to direct veneer restauration. This can only be done after measuring the possibility of preparing, elaborating and polishing the restauration.

\section{Acknowledgments}

The authors declare no conflict of interest related to this study. There are no conflicts of interest and no financial interests to be disclosed. 


\section{REFERENCES}

1. Rosenstiel SF, Rashid RG. Public preferences for anterior tooth variations: a web-based study. J Esthet Restor Dent. 2002;14(2):97-106.

2. Paolucci B, Calamita M, Coachman C, Gürel G, Shayder A, Hallawell P. Visagism: the art of dental composition. Quintessence Dent Technol. 2012;35:187-200.

3. Maier B. Aesthetic guidelines for natural-looking dentures. Cosmetic Dentistry 2011;4:14-17.

4. LeSage BP, Dalloca L. Approaches to smile design. Mathematical to artistic interpretation. J Cosmet Dent. 2012;28(1):127. 147.

5. Dietschi D. Free-hand composite resin restorations: a key to anterior aesthetics. Pract Periodontics Aesthet Dent. 1995;7(7):15-25; quiz 27.

6. Ahmad I. Protocols for predictable aesthetic dental restorations. Oxford, UK: Blackwell Munksgaard; 2006 7. Villarroel M, Fahl N, De Sousa AM, De Oliveira OB Jr. Direct esthetic restorations based on translucency and opacity of composite resins. J Esthet Restor Dent. 2011;23(2):7387.

8. Azzopardi N, Moharamzadeh K, Wood DJ, Martin N, van Noort R. Effect of resin matrix composition on the translucency of experimental dental composite resins. Dent Mater. 2009;25(12):1564-1568.
9. Ahmad I. Crafted Restorations - Shade matching with resin based composites. J Cosmet Dent. 2013;29(1):43-50. 10. Milnar J,Wohlberg J. Direct Resin Veneers. J Cosmet Dent. 2013;29(1):110-111.

11. Barakah HM, Taher NM. Effect of Polishing systems on stain susceptibility and surface roughness of nanocomposite resin material. J Prosthet Dent. $2014 ; 112(3): 625-631$. 12. Peyton JH. Finishing and polishing techniques: direct composite resin restorations. Pract Proced Aesthet Dent. 2004; 16(4):293-298; quiz 300.

13. Scoble HO, White SN. Compound complex curves: the authentic geometry of esthetic dentistry. J Prosthet Dent. 2014;111(6):448-454.

14. Gürel G, The science and art of porcelain laminate veneers. Berlin, Germany: Quintessence Pub; 2003. p 87.

15. Frese C, Schiller P, Staehle HJ, Wolff D. Recontouring teeth and closing diastemas with direct composite buildups: a 5-year follow-up. J Dent. 2013;41(11):979-985.

16. Garcia-Godoy F, Krämer N, Feilzer AJ, Frankenberger R. Long-term degradation of enamel and dentin bonds: 6-year results in vitro vs. in vivo. Dent Mater. 2010;26(11):1113-1118. 17. Gresnigt MM, Kalk W, Ozcan M. Randomized controlled split-mouth clinical trial of direct laminate veneers with two micro-hybrid resin composites. J Dent. 2012;40(9):766-775.

\section{Zoltán MISKOLCZY \\ DMD, clinical dentist \\ Department of Prosthodontics, Faculty of Dentistry Semmelweis University, Budapest, Hungary}

Dr. Zoltán Miskolczy graduated from the Faculty of Dentistry, Semmelweis University, Budapest, Hungary in 2012. Later on he started working for the Department of Prosthodontics. He has always focused on aesthetic dentistry especially on the direct and indirect veneers so as to constantly enhance his knowledge and learn new techniques.

\section{Orestions}

\section{In which case can you choose direct veneers?}

$\square$ a. Hypocalcification;

b. Abrasion;

c. Missing teeth;

d. Disathema closure.

\section{It's typical of a young frontal teeth:}

$\square$ a. HALO effect;

b. Visible perikymata;

c. Surface grooves;

$\checkmark$ d. Mamelons.

\section{A direct restoration is needed to}

$\square$ a. Diagnostic waxwork;

$\square$ b. Porcelain layering;

c. Intraoral mock-up;

d. Composite layering.

\section{The step of direct veneer implementation:}

$\square$ a. Teeth preparation;

$\square$ b. Precision Impression;

c. Conditioned the surface by means of total etch technology ( $37 \%$ orthophosphoric acid);

$\square$ d. Composite layering. 